\title{
An elderly woman presents with a "Transient Ischemic Attack”: A curable cause
}

\begin{abstract}
We report a 78-year-old female who presented to the Emergency department after a 10-minute episode of transient ischemic attack (TIA)-like symptoms of right side sensorimotor deficit, presumably due to a left carotid artery ischemia syndrome, only to be found surprisingly to have bilateral multifocal acute infarcts of cardioembolic pattern by brain magnetic resonance imaging, and, even more surprisingly, to have a rare, but curable embolic source from a large left atrial myxoma. This case report emphasizes the importance of following existing guidelines to timely and thoroughly investigate the potential management-changing causes for all TIA patients. Rare but curable causes of stroke or TIA in young adults such as cardiac myxoma can also occur in the elderly patient population.
\end{abstract}

Keywords: transient ischemic attack; ischemic stroke; myxoma; cardiac tumor; echocardiography
Volume II Issue I - 202I

\author{
Catherine A Kronfol,' Shashank Shekhar,' \\ Juebin Huang ${ }^{2}$ \\ 'Class of 2021, School of Medicine, University of Mississippi \\ Medical Center, USA \\ ${ }^{2}$ Department of Neurology, University of Mississippi Medical \\ Center, USA
}

\begin{abstract}
Correspondence: Dr. Juebin Huang, MD, PhD, Associate professor, Department of Neurology, University of Mississippi Medical Center, 2500 N. State Street, Jackson, MS, 392 16. Tel 60I-984-5500; Fax 60I-984-5503; Email jhuan@umc.edu
\end{abstract}

Received: Februry 15, 2020 | Published: Februry 24, 2021
Abbreviations: CVE, cerebrovascular events; CSE, cardiac source of embolism

\section{Case description}

\section{Initial evaluation}

A 78-year-old female, a former smoker with a history of mild Alzheimer's disease and hypertension presented to the emergency department (ED) after a transient episode of numbness and tingling of her right upper and lower extremity and weakness in her right lower extremity while walking outside. Her symptoms resolved within 10 minutes. She denied any vision change, facial droop, or speech difficulty. There was no chest pain, shortness of breath, or palpitations. Physical examination in ED was significant for elevated blood pressure to $177 / 73 \mathrm{mmHg}$, but other vital signs and general examination were normal. On neurological examination, patient was awake, alert, and oriented to person, place, and time. Speech and language were intact. There was no cranial nerve deficit, motorsensory deficit, or dysmetria. Gait was normal.

Routine labs were all normal except for a glucose level of 145 $\mathrm{mg} / \mathrm{dl}$. Electrocardiogram (EKG) was normal. Head computerized tomography (CT) reported no acute intracranial abnormality. Transient ischemic attack (TIA) in the the left internal carotid artery (ICA) vascular distribution was suspected. $\mathrm{ABCD}^{2}$ score was 4 points. CT angiogram of head and neck was only significant for focal moderate stenosis (approximately $60 \%$ ) at the origin of the right ICA. Hospital admission for further work-up was offered. Patient initially was reluctant to stay, but was convinced to come in after a lengthy discussion of the potential risks of future TIA/stroke recurrence.

\section{Hospital course}

During her hospital stay, a brain magnetic resonance imaging (MRI) was obtained which revealed multiple punctate acute infarcts in bilateral frontal lobes, parieto-occipital lobes, and left cerebellar hemisphere (Figure 1). As the MRI findings were highly suggestive of cardioembolic stroke, a transthoracic echocardiogram was then performed which revealed a large, highly mobile mass attached to the interatrial septum that prolapsed into left ventricle during diastole.
A transesophageal echocardiogram confirmed the left atrial mass (Figure 2). The patient underwent a successful surgical resection of the left atrial mass on hospital day 4, which was pathologically confirmed as a $4.0 \times 2.5 \times 1.9 \mathrm{~cm}$ cardiac myxoma. She was discharged on postoperative day 6 to home with asprin and atorvastatin for secondary stroke prevention. She has followed up in clinic for two years without recurrence of stroke.

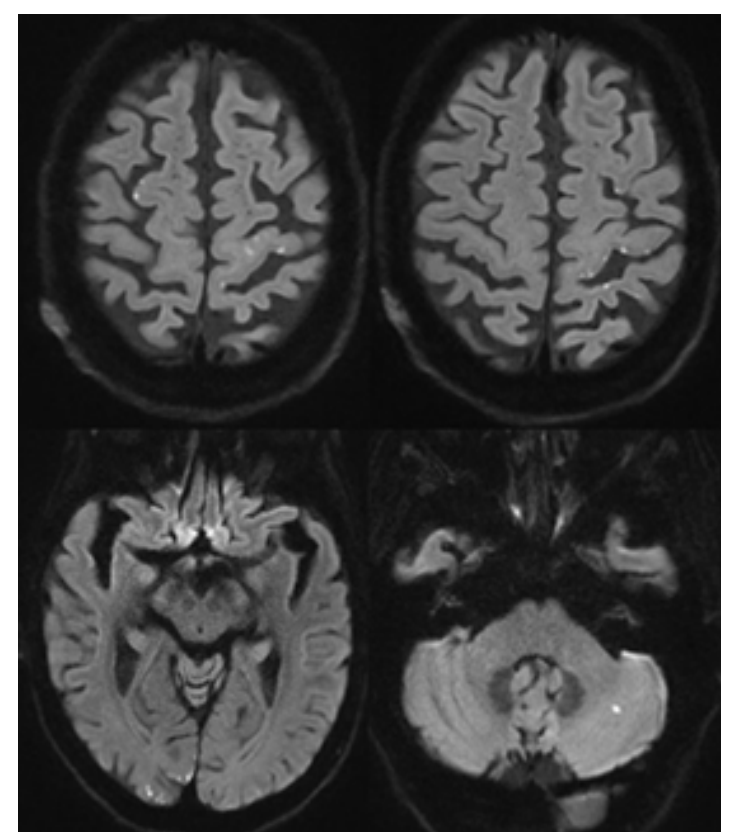

Figure I MRI diffusion-weighted imaging revealing multiple punctate infarcts in bilateral frontal lobes, parieto-occipital lobes, and left cerebellar hemisphere.

\section{Discussion}

TIA and stroke represent two ends of an ischemic continuum from the pathophysiological mechanism perspective. The distinction between TIA and ischemic stroke has become less important in recent years because many of the preventative approaches and clinical management are applicable to both. Research has shown that up to 
$50 \%$ of patients with TIA-like presentation had a detectable infarct on MRI. There is up to a $20 \%$ risk of recurrent stroke in the 90 days following a TIA with the highest risk in the first 2 days. Up to $80 \%$ of this risk is preventable by means of immediate assessment and treatment. ${ }^{1-3}$

However, compared to stroke, diagnostic certainty is often much lower with TIA and it can be a challenge even for experienced vascular neurologists. Prevention of future stroke from TIA requires identification of the underlying mechanism, as optimal prevention strategies vary based on the causes. TIA evaluation should always include proving the vascular origin of the symptoms, excluding an alternative nonischemic etiology (TIA/stroke mimics), identifying prognostic outcome categories, and determining the underlying vascular mechanism (i.e., large-vessel atherothrombotic, cardioembolic, small-vessel, cryptogenic, and uncommon subtypes such as vascular dissection, vasculitis, etc. .). ${ }^{2,3}$

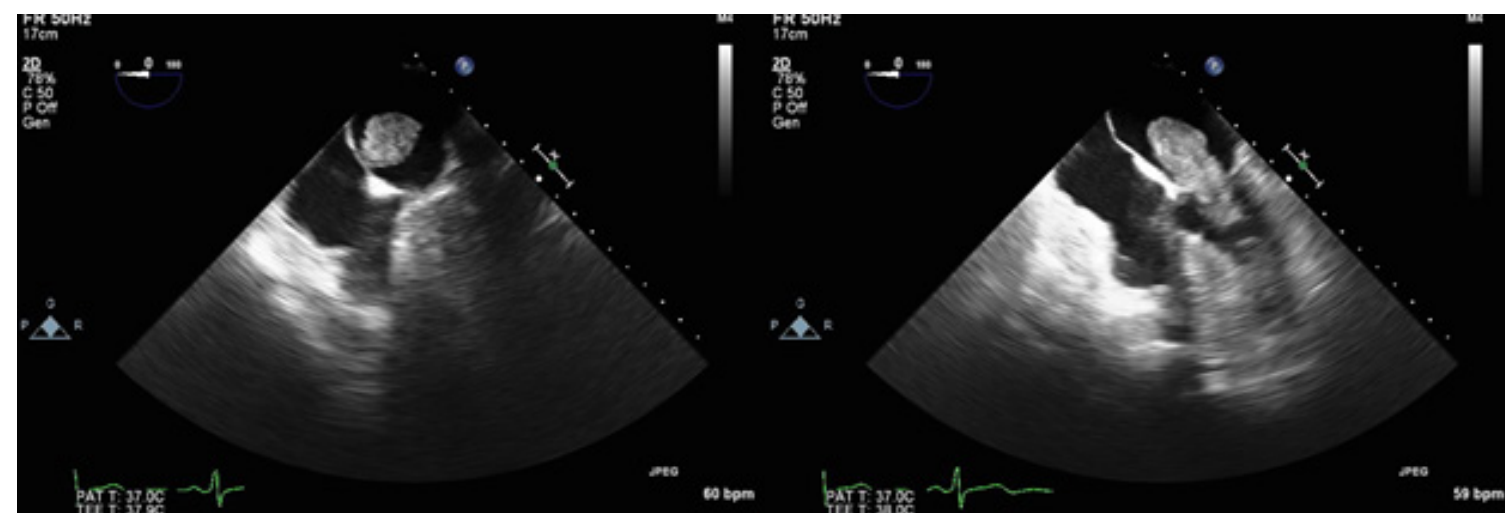

Figure 2 Transesophageal echocardiogram revealing a large, highly mobile mass attached to the interatrial septum that prolapses into the left ventricle during diastole.

Established guidelines recommend that all patients with suspected TIA should undergo prompt and comprehensive investigations just like acute stroke. TIA patients who present within 72 hours and have an $\mathrm{ABCD}^{2}$ score $>3$ are required to be hospitalized for work-up including MRI brain with diffusion sequences, cervical and intracranial vessel imaging, and EKG. Prolonged cardiac monitoring and echocardiogram are indicated for patients whose vascular etiology are unclear. ${ }^{1-3}$ However, in practice, patients with transient or minor neurologic symptoms presenting to the ED are less likely to be investigated as comprehensively as patients presenting with stroke. ${ }^{4}$ Patients with TIA are more likely to be evaluated using a rapid triage protocol, in which urgent brain and vascular imaging are obtained, and those felt to be low risk are discharged. Although outpatient follow-up for more thorough work-up is usually arranged per protocol, a significant portion of patients do not show up to the clinic or the work-up may be delayed due to limited access. A recent study suggested that even patients with TIA traditionally considered low risk carry a substantive risk of a recurrent stroke. The rate of radiologically confirmed acute stroke using early MRI was $13.5 \%$ among participants with suspected low-risk TIA or minor stroke. The implication of this finding is that if the diagnosis of TIA or minor stroke is considered, even with lowrisk features, early investigation such as MRI must be completed in a timely manner so appropriate intervention for secondary intervention can be initiated. ${ }^{4}$ Moreover, identifying management-changing potential sources of TIA, such as a cardiac source of embolism (CSE), requires structural cardiac imaging and prolonged cardiac rhythm monitoring, which often cannot be obtained in the ED or in outpatient settings timely. Thus, such investigations are often missed. In a recent study, atrial fibrillation (AF) was found to be present in $18.6 \%$ of patients with TIA, and the proportion of AF increased with age, reaching $32.9 \%$ in TIA patients $\geq 85$ years. ${ }^{5}$ In another study, a potential structural cardiac abnormality that can lead to CSE was identified in $10 \%$ of TIA patients by echocardiogram. ${ }^{6}$

Our patient presented to ED with a 10-minute episode of TIA-like symptoms of right sided sensorimotor deficit, presumably due to a left carotid artery ischemia syndrome, only to be found surprisingly to have acute infarcts of cardioembolic pattern by MRI brain, and, even more surprisingly, to have a rare, but curable CSE from a large left atrial myxoma. Although her $\mathrm{ABCD}^{2}$ score was 4 in $\mathrm{ED}$, she was initially reluctant to be admitted to the hospital. If she was discharged from ED after the initial CT head and vascular imaging, a timely investigation including MRI brain and subsequently echocardiogram would have been significantly delayed or potentially not happened at all. This would have put her at a very high risk of recurrent stroke or other complications from the myxoma.

A cardiac myxoma is a benign primary cardiac tumor that commonly occurs in the left atrium. It is a rare cause of stroke in the young and most often presents between ages 30 and $60 .^{7,8}$ Recent research suggests that the incidence of cardiac myxoma in elderly populations has increased and this may be due to the application of newer imaging techniques and longer life expectancy. Among 153 cases of cardiac myxoma reported by Karabinis et al., $49 \%$ were over 60 years old and $17.6 \%$ were over 70 years old. Embolic events due to detached tumor tissue, disseminated thrombotic material overlying the tumor, or both occurred in $30-50 \%$ of patients with cardiac myxoma, more than $50 \%$ affecting the central nervous system and the retinal arteries. Other neurological manifestations of cardiac myxoma include intracranial hemorrhage, typically secondary to myxomatous aneurysm formation and cerebral metastases. ${ }^{7}$ In a recent study of cerebrovascular events (CVE) caused by cardiac myxoma, $61 \%$ of patients presented with ischemic stroke, $23 \%$ with TIA, and $15 \%$ with retinal ischemia. ${ }^{8}$ As one can imagine, given the limited access to cardiac imaging and the lack of awareness, cardiac myxoma presenting with TIA or retinal ischemia can be easily missed in an acute care setting.

Surgical excision is the most effective, curative treatment for cardiac myxoma with low complications and recurrence rate. Conservative treatment with antiplatelets or anticoagulants for cardiac myxoma-related CVE or as bridging-therapy to delay cardiac myxoma surgery has been proposed as an alternative approach. This approach 
is particularly useful in elderly or high risk-for-surgery patients, given the fact that open-heart surgery requires systemic anticoagulation and cardiopulmonary bypass, which can cause increased risk of intracranial hemorrhage in patients with recent stroke. However, Stefanou et al. ${ }^{8}$ argued that no alternative to cardiac surgery should be considered in patients presenting with cardiac myxoma-related CVE as $46 \%$ of their cardiac myxoma patients developed CVE while taking antiplatelets or anticoagulants, $23 \%$ of the patients developed recurrent CVE under bridging-antithrombotic-therapy prior to surgical excision, and prolonged time interval between CVE and surgery was significantly associated with more CVE recurrence. They suggested that the waiting or bridging time with antithrombotic therapy before surgery should be kept as short as possible. 2019 AHA/ASA guidelines suggest use of intravenous thrombolysis in debilitating stroke as a result of cardiac myxoma (Class $\mathrm{IIb}$ ) and there are no reported contraindications to mechanical thrombectomy. ${ }^{10}$

This case report emphasizes the importance of following existing guidelines to timely and thoroughly investigate the potential management-changing causes, and to optimize prevention strategies for all patients with suspected TIA. Rare but curable causes of stroke or TIA such as cardiac myxoma can also occur in the elderly patient population.

\section{Take home points}

1. All patients with suspected TIA should undergo timely, comprehensive investigations, just like an acute stroke, to identify underlying causes and to optimize prevention strategies.

2. Even patients with TIA traditionally considered low risk carry a substantive risk of a recurrent stroke.

3. Searching for management-changing causes such as cardiac source of embolism is equally important for TIA and stroke.

4. Rare, curable stroke causes in young can also occur in elderly.

\section{Acknowledgements}

None.

\section{Sources of funding}

None.

\section{Conflicts of interest}

The authors declere no Conflicts of interest.

\section{References}

1. Amarenco P. Transient Ischemic Attack. $N$ Engl $J$ Med. 2020;382(20):1933-1941.

2. Easton JD, Saver, JL, Albers GW, et al. Definition and evaluation of transient ischemic attack. Stroke. 2009;40(6):2276-2293.

3. Kernan WN, Ovbiagele B, Black HR, et al. Guidelines for the Prevention of Stroke in Patients with Stroke and Transient Ischemic Attack. Stroke. 2014;45(7):2160-2236.

4. Coutts SB, Moreau F, Asdaghi N, et al. Rate and Prognosis of Brain Ischemia in Patients with Lower-Risk Transient or Persistent Minor Neurologic Events. JAMA Neurology. 2019;76(12):1439-1445.

5. Buchwald F, Norrving B, Petersson J. Atrial Fibrillation in Transient Ischemic Attack Versus Ischemic Stroke. A Swedish Stroke Register (Riksstroke) Study. Stroke. 2016;47(10):2456-2461.

6. Wilson CA, Tai W, Desai JA, et al. Diagnostic Yield of Echocardiography in Transient Ischemic Attack. J Stroke Cerebrovasc Dis. 2016;25(5):1135-1140.

7. Wen X, Chen Y, Yu L, et al. Neurological manifestations of atrial myxoma: A retrospective analysis. Oncology Letters. 2018;16(4):46354639.

8. Stefanou ML, Rath D, Stadler V, et al. Cardiac Myxoma and Cerebrovascular Events: A Retrospective Cohort Study. Frontier in Neurology. 2018;9:823.

9. Karabinis A, Samanidis G, Khoury M, et al. Clinical presentation and treatment of cardiac myxoma in 153 patients. Medicine (Baltimore). 2018;97(37):e12397.

10. Powers WJ, Rabinstein AA, Ackerson T, et al. Guidelines for the Early Management of Patients With Acute Ischemic Stroke: 2019 Update to the 2018 Guidelines for the Early Management of Acute Ischemic Stroke. Stroke. 2019;50:e344-e418. 